\title{
How can the gut microbiota affect immune recovery in HIV-infected individuals?
}
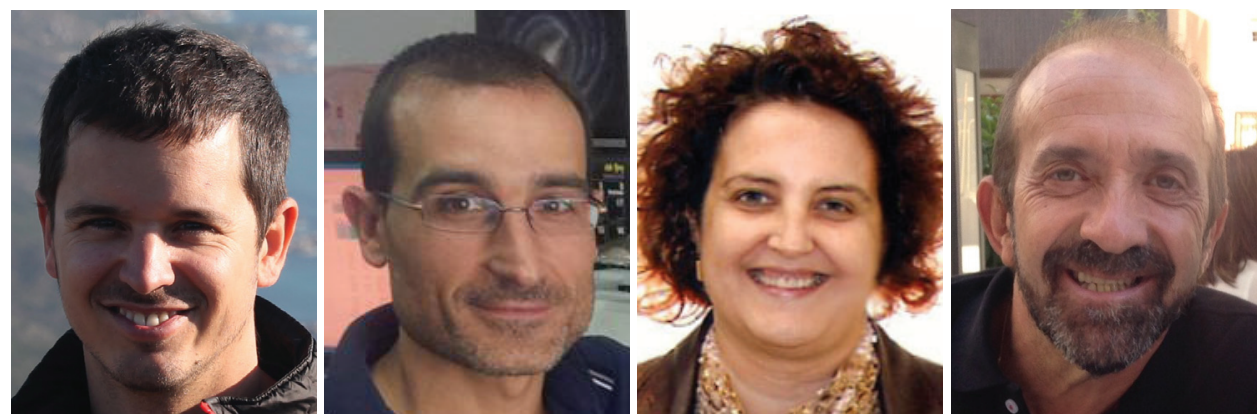

Sergio Serrano-Villar, ${ }^{* 1}$ Manuel Ferrer², María José Gosalbes ${ }^{3,4} \&$ Santiago Moreno ${ }^{1}$

First draft submitted: 11 December 2016; Accepted for publication: 3 January 2017; Published online: 15 February 2017

The explosion of the microbiome field has shaken our understanding of the pathogenesis of a number of conditions, including HIV disease, in which participation of intestinal bacteria in the course of the disease was far from being suspected. The increasingly appreciated implications in human physiology, including nutrition, metabolism and immunity have open new avenues also in HIV research. Persistence of immune defects, such as sustained activation of the innate and adaptive immunity, during otherwise effective antiretroviral therapy (ART) remains an important clinical challenge, since it contributes to an increased risk of mortality and no therapeutic strategy to date has proved ability to fully reverse these defects. Given the ability of the microbiota to instruct the immune system and control the inflammatory response, expectations about the potential usefulness of targeting the microbiota with interventions to improve the immune recovery and dampen inflammation during HIV infection are high.

Recent works have underlined that HIV-infected individuals harbor a distinct microbiota - the so-called HIV-associated dysbiosis. The ongoing hypothesis that some bacteria might evolve in response to HIV infection to help controlling disease progression begins to take shape. In this line, there is emerging consensus that disturbances in the gut microbial ecology during HIV infection correlate with chronic immune defects and inflammation [1]. For example, microbial diversity in feces of HIV-infected patients seems to inversely correlate with biomarkers of microbial translocation and monocyte

\section{KEYWORDS}

- HIV • microbiota $\bullet$ metabolomics - immune recovery $\bullet$ inflammation

\section{“Untangling the host-microbiota interactions influencing health and disease has proved to be a formidable challenge, and understanding the effects of therapies modulating the microbiota structure in complex diseases, such as HIV infection might add an additional level of difficulty."}

'Department of Infectious Diseases, Hospital Universitario Ramón y Cajal. Facultad de Medicina, Universidad de Alcalá 
"We have just begun to understand which

microbial and metabolic

changes are partially

random consequences of

HIV infection,

environmental factors and the use of chronic

antiretroviral therapy, and which are the potential

causes, as well as their associations in the context of immune recovery." activation [2], and changes in mucosa-associated bacteria correlate with inflammation and T-cell activation $[3,4]$.

Most studies in the field of HIV infection and microbiota have largely relied on the sequencing of $16 \mathrm{~S}$ ribosomal gene to infer the composition of the total bacterial communities. Using this methodology, we know that a distinct composition of the microbiota is established in HIVinfected patients, in which at least 54 nonredundant genera belonging to five main different phyla (Bacteroidetes, Firmicutes, Proteobacteria, Spirochaetes and Verrucomicrobia) are significantly altered. Bacteria belonging to the Enterobacteriaceae, Erysipelotrichaceae and Veillonellaceae families have been also found as biomarkers in HIV-infected patients. Whether this dysbiosis is elicited by HIV infection itself or rather is a consequence of differences in lifestyle, comorbidities and the use of chronic ART remains yet to be elucidated.

However, recent data suggest that the total bacterial composition is not representative of the active fraction of the microbiota [5-7]. Indeed, the analysis of $16 \mathrm{~S}$ ribosomal gene analysis does not differentiate between dormant, dead or quiescent bacteria, so it is not useful to establish which genera are in an active and potentially active state. Their identities and abundances can be estimated by monitoring the $16 \mathrm{~S}$ ribosomal genes generated from cDNA (so-called RNA-seq), and by the taxonomic binning of sequences from genes found to be expressed in the metatranscriptome and proteins found to be synthesized in the metaproteome, because they are transcriptionally active. Using these methodologies, we have found that not all bacterial groups in our gut equally react to HIV infection, and that bacteria belonging to the Acidaminococcaceae, Prevotellaceae, Bacteroidaceae, Desulfovibrionaceae, Enterobacteriaceae, Erysipelotrichaceae, Eubacteriaceae, Lachnospiraceae and Veillonellaceae families are among those that become transcriptionally active during HIV infection [7].

The next logical step is to infer the metabolic consequences associated to the alterations in our microbes during HIV infection. However, recent observations that different intestinal populations are metabolically redundant indicate that taxonomic diversity is hardly relevant for inferring functional traits $[8]$. Thus, we have begun to apply metagenomics and metatranscriptomic studies based on high-throughput DNA and cDNA sequencing to quantify alterations in the content and expression level of microbial genes to provide deeper insight into the potential functional pathways involved in HIV immunopathogenesis. The first study in HIV-infected individuals using shotgun DNA sequencing found altered metabolic capabilities, with enrichment of genes involved in several pathogenic processes, such as lipopolysaccharide biosynthesis and bacterial translocation and depletion of genes involved in amino acid metabolism and energy processes [5]. More recently, a study in a larger cohort of HIV-infected individuals has found that HIV infection is associated with low bacterial gene richness, particularly in subjects with poor immune recovery [9]. A higher bacterial richness was found here indicative of greater abundance of butyrate-producing bacteria. Butyrate, a short-chain fatty acid (SCFA), is an end product of bacterial anaerobic fermentation of dietary fiber and might influence health by different mechanisms. For example, butyrate is the preferential energy source of gut epithelial cells [10], activates human genes via histone deacetylase inhibition [11] and promotes immunotolerance to commensal bacteria via downregulation of lipopolysaccharideinduced proinflammatory mediators [12]. The abundance of Faecalibacterium prausnitzii, a dominant butyrate-producer in the healthy gut, is lower in inflammatory bowel disease [13,14]. In HIV-infected individuals we have found a dramatic depletion of $F$. prausnitzii, as well as an altered SCFA profile, indicating a potential role for the SCFA-synthesis pathway in HIV immunopathogenesis [6].

Other relevant opportunity offered by the metagenomics' approach is to identify sequencepredicted metabolic alterations that are a reflection of the human-microbiota axis. A fascinating mechanism of bacterial influence on a human proinflammatory pathway was first described in a seminal work by Vujkovic-Cvijin et al., showing that a number of taxa belonging to the HIV-associated dysbiotic communities encoded the genetic machinery that performs the same tryptophan catabolism as human indoleamine2,3-dioxygenase-1 (IDO-1) [3]. This enzyme is constitutively expressed in macrophages and dendritic cells, and its activation results in the production of the immunosuppressive kynurenine derivatives, which impair the mucosal immunity, resulting, among other defects, in loss 
of Th17 cells. Indeed, induction of the IDO-1 is associated with impaired mucosal immunity, bacterial translocation and mortality [15]. Strikingly, tryptophan can be also catabolized by dysbiotic bacteria, yielding to the production of the same immunosuppressive metabolites [3]. These data are in agreement with a recent study in which we characterized the changes of gut bacteria metabolism in HIV-infected subjects, as compared with other diseases. We found that 3-hydroxyanthranilate, a subproduct of the kynurenine pathway, accumulates in the microbiota of HIV-infected individuals. Lactobacillus, a genus widely considered as beneficial for the homeostasis of the gut immune system, has been found to be depleted in simian immunodeficiency virus (SIV)-infected macaques and early HIV-infected patients and strongly correlates with IDO-1 activity and Th17 loss. Of note, supplementation of Lactobacillus spp. in SIV-infected macaques significantly inhibited IDO-1 catabolism of tryptophan [16], arguing that the bacterial induction of the kynurenine pathway can be attenuated with dietary probiotic supplementation. So, it is likely that some components of the HIV-affected dysbiosis affect immunity via emulation of the human kynurenine pathway and that probiotics might attenuate this pathogenic circle. Other species typically dominant in HIV-infected individuals seem to induce the production of proinflammatory cytokines when cultured with mononuclear cells obtained from peripheral blood or the intestinal lamina propria [17]. Hence, several taxa from the $\mathrm{HIV}$-associated microbiota appear to contribute to local and systemic inflammation by different mechanisms.

Therefore, characterizing the total bacterial composition and sequence-based predictions from genes being sequenced and annotated is useful, but not sufficient, for the purpose of linking microorganisms to genes and proteins, and subsequently to functions and the host during HIV infection [18]. This can be revealed by integrating data not only from the abundance of dead, quiescent or active species and predicted altered gene contents, but also from altered content and fluxes of their metabolic products, that is their proteins and metabolites [8]. Examination of microbial protein (through metaproteomics) and metabolite (through metabolomics) contents and fluxes can reveal whether metabolic alterations are a reflection of the differences observed at the level of population structure.
Also, most of the biological effects attributed by the microbiota are driven by their metabolites (i.e., the effector molecules) and this is why their analysis is now considered essential to obtain a global picture of microbiota composition and microbiome function from a pathogenic perspective. Thus, we have recently analyzed in HIVinfected patients the proteins and metabolites that are actively synthesized and absorbed, metabolized and produced, respectively, by the microbiota as well as in the bloodstream [7]. We found that HIV infection is associated to specific protein and metabolite profiles. Also, distinct profiles characterize the HIV-infected patients with different immune recovery levels. Through proteomics and metabolomics we found that a number of active bacteria (e.g., Bifidobacteria) control epithelial cell turnover, and their activation in patients with immune recovery prevent intrinsic defects in the mucosa. In striking contrast, subjects with poor immune recovery showed cleavage of sialic and dolichol components from epithelial cells by gut bacteria, presumably causing abnormal glycan composition in enterocytes and fueling microbial translocation. This allowed that proinflammatory cytokines accumulated in the gut environment (i.e., fecal fluid) rather than within bacteria, likely promoting local inflammation and limiting immune recovery. More interestingly, bacteria from the Succinivibrionaceae family, and possibly Erysipelotrichaceae, acted as inflammatory buffers by accumulating proinflammatory molecules, such as arachidonic acid and its derivative leukotriene-B4. They also may accumulate human molecules with antiviral activity, such as cannabinoid oleamide and biliverdin. As such bacteria become transcriptionally active in patients with high immune recovery level, it is plausible that gut bacteria can contribute to health recovery by inhibiting viral replication, stimulating the immune system and ultimately reducing inflammation. The mechanisms by which bacteria sense the immune status to become transcriptionally active deserve further investigation.

Studies with therapeutic interventions will help to settle the debate whether HIV-associated microbiota is simply a consequence of lifestyle factors, HIV infection and its treatment or if it actually plays a relevant role in the dynamics of $\mathrm{T}$ cells and inflammatory markers during ART. In untreated HIV-infected individuals, interventions with probiotics and/or prebiotics

\author{
“Recent findings applying \\ complementary OMICS' \\ techniques have revealed \\ that our microbes are not \\ mere actors being \\ influenced when \\ confronted to the HIV \\ infection, but rather are \\ playing an active role in \\ disease progression.”
}


have been shown to contribute to maintain the CD 4 counts over a longer period [19-21]. In SIVinfected macaques beginning ART, a synbiotic treatment (a mixture of prebiotics and probiotics) improved the frequency and functionality of gastrointestinal antigen-presenting cells, enhanced reconstitution and functionality of $\mathrm{CD} 4^{+} \mathrm{T}$ cells, and reduced fibrosis of lymphoid follicles in the colon [22]. In two recent placebo-controlled trials with treated HIV-infected individuals, a probiotic intervention caused a significant reduction in the inflammatory predictors of mortality IL-6 and D-dimers, which in turn was associated with increases in Bifidobacteria and Lactobacilli $[23,24]$. In a recent pilot study, we have found that a short dietary supplementation with prebiotics attenuated HIV-associated dysbiosis, yet the microbiota of ART-treated subjects was found more resilient - a term used in ecology to refer to the capacity to absorb perturbations. We observed that increases in the abundance of Faecalibacterium and Lachnospira strongly correlated with moderate but significant increases of butyrate production and amelioration of inflammatory biomarkers soluble CD14 and high-sensitivity $\mathrm{C}$ reactive protein, especially among ART-naive individuals. So, while we found that bacterial butyrate synthesis pathway seems a viable target for interventions, it is likely that more energetic interventions are required to significantly affect the gut microbiota of ART-experienced. Fecal microbiota transplantation has gained acceptance for treatment of Clostridium diffcile infection, and has been evaluated via colonoscopy in HIV-infected individuals, showing a limited ability to restore the microbiota [25]. New approaches, including preconditioning treatment with antibiotics to improve bacterial donor's engraftment or repeated fecal microbiota transplantation are currently under investigation.

Untangling the host-microbiota interactions influencing health and disease has proved to be a formidable challenge, and understanding the effects of therapies modulating the microbiota structure in complex diseases, such as HIV infection might add an additional level of difficulty. We have just begun to understand which microbial and metabolic changes are partially random consequences of HIV infection, environmental factors and the use of chronic ART, and which are the potential causes, as well as their associations in the context of immune recovery. Recent findings applying complementary OMICS' techniques have revealed that our microbes are not mere actors being influenced when confronted to the HIV infection, but rather are playing an active role in disease progression. Therefore, it is likely that the analysis of dormant, dead, quiescent and active bacteria (metataxonomic analysis and metagenomics) and their molecular products, including genes (metatranscriptomes), proteins (metaproteomes) and metabolites (metabolomes), will help revealing what species become transcriptionally activated and affect more profoundly immune recovery and define the critical active pathways implicated.

\section{Financial \& competing interests disclosure}

The authors have no relevant affiliations or financial involvement with any organization or entity with a financial interest in or financial conflict with the subject matter or materials discussed in the manuscript. This includes employment, consultancies, honoraria, stock ownership or options, expert testimony, grants or patents received or pending, or royalties.

No writing assistance was utilized in the production of this manuscript.

\section{References}

1 Dillon SM, Frank DN, Wilson CC. The gut microbiome and HIV-1 pathogenesis: a two way street. AIDS 30, 2737-2751 (2016).

2 Nowak P, Troseid M, Avershina E et al. Gut microbiota diversity predicts immune status in HIV-1 infection. AIDS 29, 2409-2418 (2015).

3 Vujkovic-Cvijin I, Dunham RM, Iwai S et al. Dysbiosis of the gut microbiota is associated with HIV disease progression and tryptophan catabolism. Sci. Transl. Med. 5, $193 \mathrm{ra91}$ (2013).

4 Dillon SM, Lee EJ, Kotter CV et al. An altered intestinal mucosal microbiome in
HIV-1 infection is associated with mucosal and systemic immune activation and endotoxemia. Mucosal Immunol. 7, 983-994 (2014).

5 Vázquez-Castellanos JF, Serrano-Villar S, Latorre A et al. Altered metabolism of gut microbiota contributes to chronic immune activation in HIV-infected individuals. Mucosal Immunol. 8, 760-772 (2015).

6 Serrano-Villar S, Vázquez-Castellanos JF, Vallejo A et al. The effects of prebiotics on microbial dysbiosis, butyrate production and immunity in HIV-infected subjects. Mucosal Immunol. doi:10.1038/mi.2016.122 (2016) (Epub ahead of print).
7 Serrano-Villar S, Rojo D, Martínez-Martínez $\mathrm{M}$ et al. Gut bacteria metabolism impacts immune recovery in HIV-infected individuals. EBioMedicine 8, 203-216 (2016).

8 Moya A, Ferrer M. Functional redundancyinduced stability of gut microbiota subjected to disturbance. trends microbiol. Trends Microbiol. 24, 402-413 (2016).

9 Guillén Y, Noguera-Julian M, Rivera J et al. Human gut microbial gene richness correlates with HIV infection. Presented at: 2nd International Workshop on Microbiome in HIV Pathogenesis, Prevention and Treatment. Bethesda, MD, USA, 17-18 November 2016. 
10 Bik EM. Composition and function of the human-associated microbiota. Nutr. Rev. 67(Suppl. 2), S164-S171 (2009).

11 Imai K, Ochiai K, Okamoto T. Reactivation of latent HIV-1 infection by the periodontopathic bacterium Porphyromonas gingivalis involves histone modification. J. Immunol. 182, 3688-3695 (2009).

12 Chang PV, Hao L, Offermanns S, Medzhitov R. The microbial metabolite butyrate regulates intestinal macrophage function via histone deacetylase inhibition. Proc. Natl Acad. Sci. USA 111, 2247-2252 (2014).

13 Seekatz AM, Aas J, Gessert CE et al. Recovery of the gut microbiome following fecal microbiota transplantation. MBio 5 , e00893-14 (2014).

14 Machiels K, Joossens M, Sabino J et al. A decrease of the butyrate-producing species Roseburia hominis and Faecalibacterium prausnitzii defines dysbiosis in patients with ulcerative colitis. Gut 63, 1275-1283 (2014).

15 Routy J-P, Mehraj V, Vyboh K, Cao W, Kema I, Jenabian M-A. Clinical relevance of kynurenine pathway in HIV/AIDS: an immune checkpoint at the crossroads of metabolism and inflammation. AIDS Rev. 17, 96-106 (2015).
16 Vujkovic-Cvijin I, Swainson LA, Chu SN et al. Gut-resident Lactobacillus abundance associates with IDO1 inhibition and Th17 dynamics in SIV-infected macaques. Cell Rep. 13(8), 1-9 (2015).

17 Palmer B, Neff C, Li S, Schneider J, Campbell T, Lozupone C. HIV-associated alterations in gut microbiota are associated with increased inflammation of enteric $\mathrm{CD}^{+}$ T cells. Presented at: International AIDS Conference. Durban, South Africa, 22-26 July 2016.

18 Schroeder BO, Bäckhed F. Signals from the gut microbiota to distant organs in physiology and disease. Nat. Med. doi:10.1038/nm.4185 (2016) (Epub ahead of print).

19 González-Hernández LA, Jave-Suarez LF, Fafutis-Morris $\mathrm{M}$ et al. Synbiotic therapy decreases microbial translocation and inflammation and improves immunological status in HIV-infected patients: a doubleblind randomized controlled pilot trial. Nutr. J. 11, 90 (2012).

20 Cahn P, Ruxrungtham K, Gazzard B et al. The immunomodulatory nutritional intervention NR100157 reduced CD4+ ${ }^{+}$T-cell decline and immune activation: a 1-year multicenter randomized controlled double-blind trial in HIV-infected persons not receiving antiretroviral therapy (The BITE Study). Clin. Infect. Dis. 57, 139-46 (2013).
21 Hummelen R, Changalucha J, Butamanya NL et al. Effect of 25 weeks probiotic supplementation on immune function of HIV patients. Gut Microbes 2, 80-85 (2011).

22 Klatt NR, Canary LA, Sun X et al. Probiotic/ prebiotic supplementation of antiretrovirals improves gastrointestinal immunity in SIV-infected macaques. J. Clin. Invest. 123, 903-907 (2013).

23 Villar-García J, Hernández JJ, GüerriFernández R et al. Effect of probiotics (Saccharomyces boulardii) on microbial translocation and inflammation in HIVtreated patients: a double-blind, randomized, placebo-controlled trial. J. Acquir. Immune Defic. Syndr. 68, 256-263 (2015).

24 Stiksrud B, Nowak P, Nwosu FC et al. Reduced levels of $\mathrm{D}$-dimer and changes in gut microbiota composition after probiotic intervention in HIV-infected individuals on stable ART. J. Acquir. Immune Defic. Syndr. 70, 329-337 (2015).

25 Somsouk M, Vujkovic-Cvijin I, Montha P, Hunt PW, Lynch S, McCune JM. Safety and engraftment of fecal microbiome transplantation during treated HIV infection. Presented at: $23 r d$ Conference on Retrovirus and Opportunistic Infections. Boston, MA, USA, 2016 (Abstract 264). 